\title{
Labelled quotient graphs and topological features in crystal structures
}

\author{
Jean Guillaume Eon ${ }^{1}$ \\ ${ }^{1}$ Institute Of Chemistry, Federal University Of Rio De Janeiro, Rio De Janeiro, Brazil \\ E-mail: jgeon@iq.ufrj.br
}

The combinatorial or bond-topology of a crystal structure is defined as the respective underlying net, which is represented by a labelled quotient graph. Thus, every topological property of crystal structures can be determined from an analysis of their labelled quotient graph. Due to the finiteness of the quotient graph, the substitution is expected to turn the analysis easier.

A periodic net contains finitely many vertex and edge-lattices. A transversal is defined as a complete set of representatives of vertex and edge-lattices. A fundamental transversal is a connected transversal; a fundamental transversal can be thought of as a unit cell of a periodic net, but it is not a graph. Some edges, called half-edges, have only one end-vertex; the missing vertex is called a boundary vertex of the fundamental transversal. The quotient graph of a periodic net can be obtained from a fundamental transversal by closing every half-edge to the representative vertex of the vertex-lattice its boundary vertex belongs to. The label of the respective edge of the quotient graph corresponds to the translation vector that maps the corresponding representative vertex to the boundary vertex of the half-edge. Figure (a) shows the kagome $(\mathrm{kgm})$ net with a unit cell and a fundamental transversal in red. Figure (b) reproduces this same transversal with labels A, B and $C$ for the three vertex-lattices of the $\mathrm{kgm}$ net. After closing the four half-edges, one gets the labelled quotient graph shown in Figure (c). The label 10 assigned to edge BA, for example, means that the representative edge in the periodic net links vertex $B$ in the unit cell to vertex $A 10$, the vertex mapped by applying the translation of vector 10 to vertex $A$. In the same way, it means that the edge from this same edge-lattice incident to vertex $B m, n$ links this vertex to vertex $A m+1, n$. Non-labelled edges link vertices inside the unit cell.

Among closed paths in periodic nets rings play an important role in the classification of crystal structures. Such paths project to closed walks of the quotient graph with zero net voltage (the sum of the vector labels over the edges oriented along the path) and can be determined through the analysis of the quotient graph. Building-units of crystal structures can be finite or infinite, corresponding to one-, two- or even three-periodic subnets. Decomposing periodic nets into their building-units relies on graph-theoretical methods classified as surgery techniques. Instead, these operations can be performed on their labelled quotient graphs revealing directly topological relations. The maximum symmetry of a crystal structure is given by the group of automorphisms of the labelled quotient graph that are consistent with net voltages over the respective cycles. In modular compounds the structure and maximum symmetry of the module, i.e. its layer group, can be determined directly from the quotient graph. Partial symmetry operations between different modules are associated to automorphisms of the quotient graph that may not be consistent with net voltages over the respective cycles. These operations generate a groupoid structure.

[1] Chung S.J. et al. (1984) Acta Cryst. A40, 42-50.

[2] Eon J.-G. (2011) Acta Cryst. A67, 68-86.

[3] Eon, J.-G. (2016) Acta Cryst. A72, 268-293.

(a)

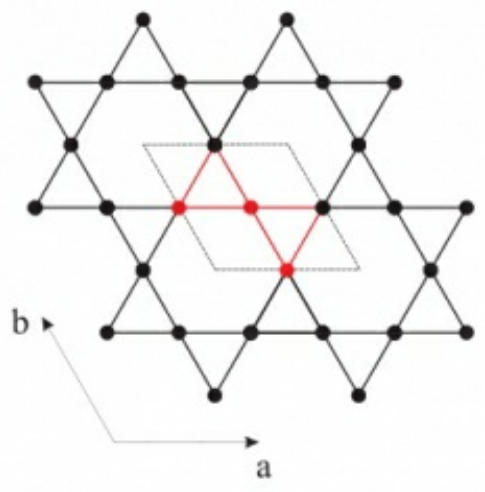

(b)

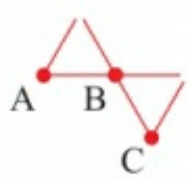

(c)

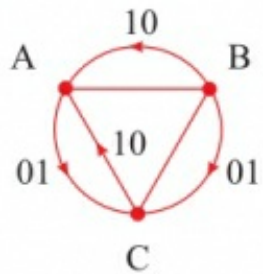

Keywords: Labelled quotient graphs, topological descriptors, symmetry 